\title{
Analysis of the Influence of NESZ Variations on Cross-Polarized Signatures of Sea Ice
}

\author{
B. Scheuchl and I. Cumming \\ Department of Electrical and Computer Engineering, University of British Columbia, \\ 2356 Main Mall, Vancouver, B.C., Canada V6T 1Z4
}

\begin{abstract}
The information content retrievable from ENVISAT ASAR cross-polarization data is evaluated with respect to sea ice monitoring. Low backscatter from sea ice and open water in combination with variations in the NESZ pose challenges for data visualization and classification. A novel classification scheme based on parallel Wishart classifiers for single and dual polarization data is introduced that allows the utilization of crosspolarized information for part of the scene.
\end{abstract}

Keywords - ENVISAT ASAR; cross-polarization data; sea ice classification

\section{INTRODUCTION}

Spaceborne single polarization SAR sensors represent the single most important data source for ice services around the world. The availability of spaceborne multi-polarization SAR data for sea ice monitoring is expected to improve the information content available to ice analysts. ENVISAT ASAR is the first satellite to provide data in two polarizations using its alternating polarization (AP) mode. The availability of continued coverage of sea ice infested areas provides a first opportunity to evaluate both the potential and limitations of such data.

ASAR AP data show two limitations with respect to operational sea ice monitoring:

- Short revisit intervals are a key requirement for operational monitoring. The $100 \mathrm{~km}$ AP swaths are too narrow compared with single polarization ScanSAR.

- Cross-polarization backscatter of sea ice is known to be relatively low for certain ice types. The Noise Equivalent Sigma Zero (NESZ) of a spaceborne system is generally higher than that of airborne systems and may reach or even exceed the backscatter level.

Dual polarization ScanSAR data with wide coverage will be available on RADARSAT-2. The higher NESZ level in satellite SARs is a result of the sensor design, which is constrained by the need to operate on a space platform. This paper evaluates the potential of the cross-polarization channel for ice type classification. Following a discussion of the NESZ level and its variations over range, a classification scheme is suggested that allows the utilization of dual-polarization information in areas where the cross-polarization channels is expected to contain useful information.

This research was funded by an NSERC CRD grant and by the BC Advanced Systems Institute.

\section{ENVISAT ASAR APM DATA}

In 2003, ESA regularly acquired ASAR AP data over Resolute $\left(74^{\circ} 42^{\prime} \mathrm{N}, 94^{\circ} 54^{\prime} \mathrm{W}\right)$, one of the official calibration sites. Combinations of co- and cross-polarization data were collected analysis results for the data set are reported in [1]. The medium resolution product (APM) was selected to allow for longer acquisition lines.

On October $18, \mathrm{VV}+\mathrm{VH}$ data were acquired in IS4 mode $\left(31^{\circ}-36.3^{\circ}\right.$ incidence). The two channels are shown in Fig. 1. Available auxiliary information is summarized in Table I.

Fig. 1 clearly shows the different information contained in the two channels. An area of open water shown in the lower half of the image is wind roughened, which affects the VV backscatter, but not VH. The latter is mainly dark for open water areas. Sea ice in the scene is mostly ice that has survived one or more summers melt (see Table I). In near range, the area just above Somerset Island appears dark in VV, which indicates grey ice under these conditions.

A variation of $\mathrm{VH}$ in range can be observed for the open water area (Fig. 1). In far range, $\mathrm{VH}$ increases visibly. A similar but less pronounced increase is present in the first third of near range. These variations are not expected for $\mathrm{VH}$ backscatter of open water but are rather the result of NESZ variations due to antenna pattern correction combined with a SNR $<0 \mathrm{~dB}$. The effect is discussed in more detail in the next section.

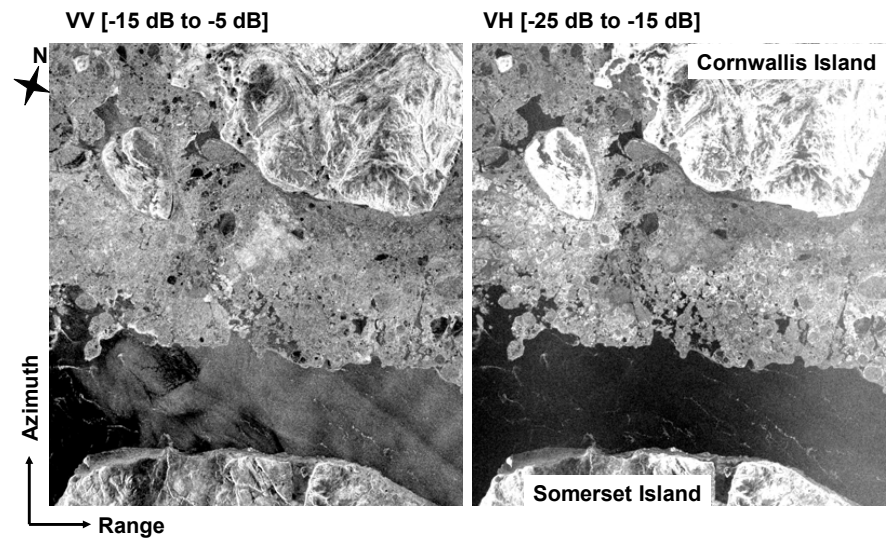

Figure 1. VV image (left) and VH image (right) of the October 18 acquisition. Note that the gray level scales for the two images differ by $10 \mathrm{~dB}$. Land shows bright in the VH image. 
TABLE I. AUXILIARY DATA

\begin{tabular}{|l|l|l|}
\hline \multicolumn{1}{|c|}{$\begin{array}{c}\text { Auxiliary } \\
\text { data }\end{array}$} & \multicolumn{1}{c|}{ Value } & \multicolumn{1}{c|}{ Comment } \\
\hline Temperature & $-4{ }^{\circ} \mathrm{C}$ & $\begin{array}{l}\text { Temperature increased compared to } \\
\text { previous and following days. }\end{array}$ \\
\hline Wind speed & $40 \mathrm{~km} / \mathrm{h}$ & $\begin{array}{l}\text { Higher wind speeds compared to previ- } \\
\text { ous and following days. }\end{array}$ \\
\hline Snowfall & $7 \mathrm{~cm}$ & Snowfall above $5 \mathrm{~cm}$ on Oct. 5 and 18. \\
\hline Ice chart & $\begin{array}{l}\text { Mostly SYI } \\
\text { and MY; } \\
\text { some Grey } \\
\text { Ice }\end{array}$ & $\begin{array}{l}\text { Ice chart for October } 18 \text { does not cover } \\
\text { the area; October 5 ice chart informa- } \\
\text { tion used instead. }\end{array}$ \\
\hline
\end{tabular}

SYI: Second Year Ice; MYI: Multi Year Ice

\section{NESZ ANALYSIS}

The NESZ and its variation in range are analyzed using a cross section of the scene in the open water area. Fig. 2 shows a range cross section with 87 azimuth lines averaged to reduce the effect of speckle. Spikes in the plot are mainly caused by ice signatures inadvertently included in the average. Crosspolarized backscatter of water and ice should have a small dependency on the incidence angle [2]. The variation observed is approximately $4 \mathrm{~dB}$ over the swath, with the highest values in far range. A drop-off in near range is present and probably caused by a change in SNR due to a signal level variation.

The likely reason for the variation of the NESZ is the high receiver noise level relative to the signal level in combination with the antenna elevation pattern correction. Fig. 3 shows the antenna elevation pattern for the scene. Elevation pattern correction is applied to compensate for low antenna gain on the beam edges, which results in raising the receiver noise.

ASAR specifications state the worst case NESZ for IS4 as $-19 \mathrm{~dB}$. These levels are not expected to be an issue for land applications. Low cross-polarized backscatter from smooth first year ice and open water may result in an SNR $<0 \mathrm{~dB}$ and thus any variations in the NESZ will affect both visual analysis and automated classification.

The potential of the VH channel for sea ice - open water separation justifies the utilization of this information. To reduce the effect of the NESZ variation over range, the utilization of the VH channel should be carefully implemented.

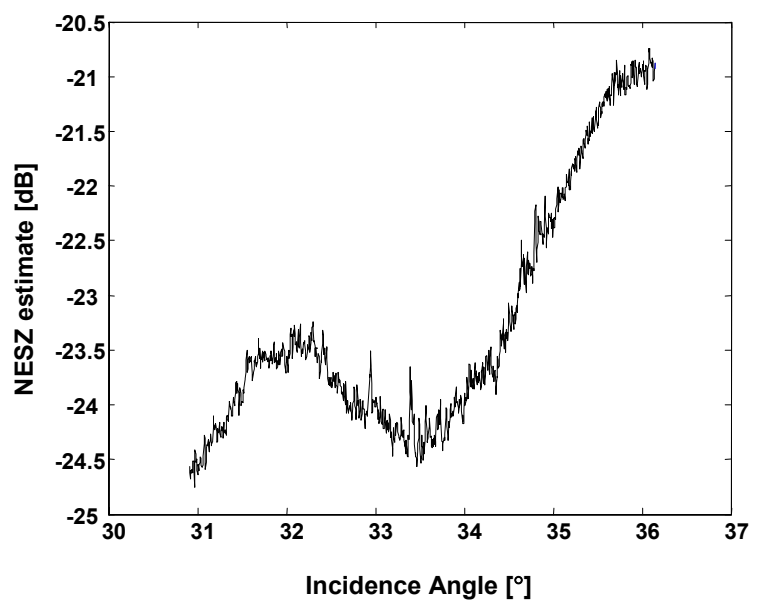

Figure 2. NESZ estimate over range. For this graph, 85 lines were averaged in azimuth in the open water region of the image.

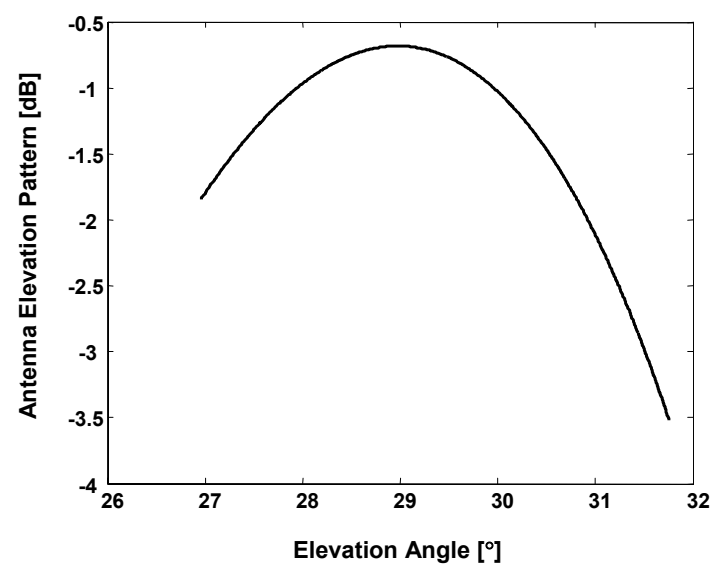

Figure 3. Antenna Elevation Pattern as provided in the ASAR data header.

\section{ClassIFICATION}

The Bayesian Wishart classifier has been shown to work for single channel as well as complex dual polarization data (i.e. partial scattering matrices) [3]. The authors also suggest an approach for detected dual polarization data, by modifying the distributions and using the magnitude of the channel correlation. Assuming that the co- and cross-polarization channels are not correlated, the Wishart classifier can be used with the detected dual polarization data [1].

Assuming the worst case NESZ for the IS4 mode of $-19 \mathrm{~dB}$, there would be little gain in using the cross-polarized channel as additional source for information. Fig. 1 shows the potential of the cross-pol information and an effort should be made to utilize this information. The variation of the NESZ can actually be used to include VH in areas of low NESZ in the classification scheme.

Fig. 4 shows the histogram of $\mathrm{VH}$ for the open water area. Based on the averaged range line, $\mathrm{VH}$ is suggested to be excluded from classification for incidence angles larger than $34.75^{\circ}$ when a low signal level is present. The resulting NESZ threshold is approximately $-22.5 \mathrm{~dB}$ thus restricting NESZ variations to about $2 \mathrm{~dB}$.

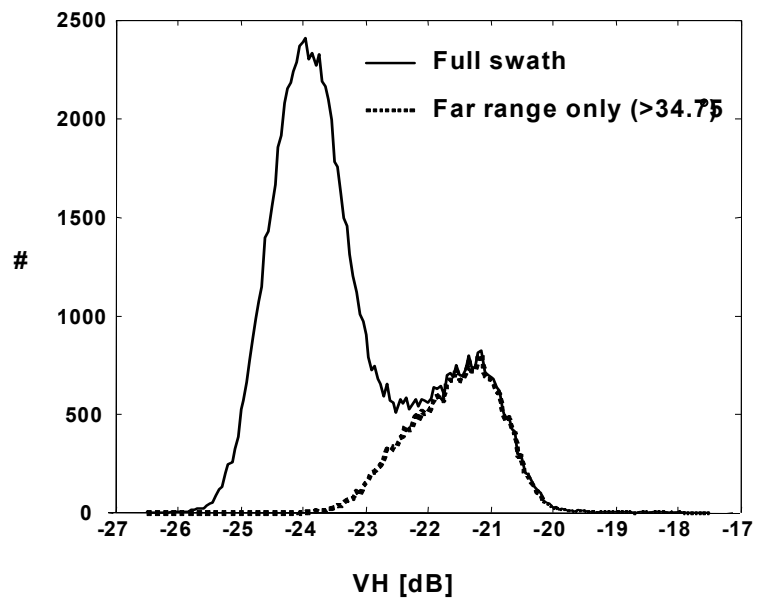

Figure 4. VH Histogram for the open water area. The dotted line shows the area proposed to be excluded from classification. 
Using this framework, a novel classification scheme can be implemented as follows:

- Use two Wishart classifiers [3] in parallel - one using the co-polarization channel only, the other one using both available channels. An iterative approach (3 iterations) is recommended.

- The two results are merged by utilizing the dual channel result in all areas where the cross-pol channel is not restricted. In areas with known high NESZ (in this case far range) and low signal level (less than $3 \mathrm{~dB}$ above the estimated noise level) the result from the copolarization only classifier is used.

- Class means for the iterations are generated for the two channels separately but always from the same (the merged) result. For the co-pol channel the entire marine area is used, whereas the cross-pol channel is restricted to certain incidence angles.

A graphical illustration of the classification scheme is presented in Fig. 5.

The scalar distance measures are very similar, for the single channel case the distance measure $\mathrm{d}_{1}$ is:

$$
d_{1}=\ln \left(\left|\mathbf{C}_{1 \mathbf{m}}\right|\right)+\operatorname{Trace}\left(\mathbf{C}_{1 \mathbf{m}} \cdot \mathbf{Z}_{1}^{-1}\right)
$$

For the dual channel case, the distance measure $\mathrm{d}_{2}$ is:

$$
d_{2}=\ln \left(\left|\mathbf{C}_{\mathbf{2 m}}\right|\right)+\operatorname{Trace}\left(\mathbf{C}_{\mathbf{2 m}} \cdot \mathbf{Z}_{2}^{-1}\right)
$$

In both cases each pixel is assigned to the class with the minimum distance, $\mathrm{d}_{1,2}$. The scalar $\mathbf{C}_{\mathbf{1 m}}=\mathrm{E}\left[\mathbf{Z}_{\mathbf{1}} \mid \omega_{\mathrm{m}}\right]$ is the mean copolarized backscatter for class $\omega_{\mathrm{m}}$. The matrix $\mathbf{C}_{\mathbf{2 m}}=\mathrm{E}\left[\mathbf{Z}_{\mathbf{2}} \mid \omega_{\mathrm{m}}\right]$ is the mean $2 \times 2$ reduced covariance matrix for class $\omega_{\mathrm{m}}$ with zero off diagonal elements. $\mathbf{Z}_{\mathbf{1}}$ and $\mathbf{Z}_{\mathbf{2}}$ are the co-pol information and the $2 \times 2$ reduced covariance matrix of the pixel to be classified.

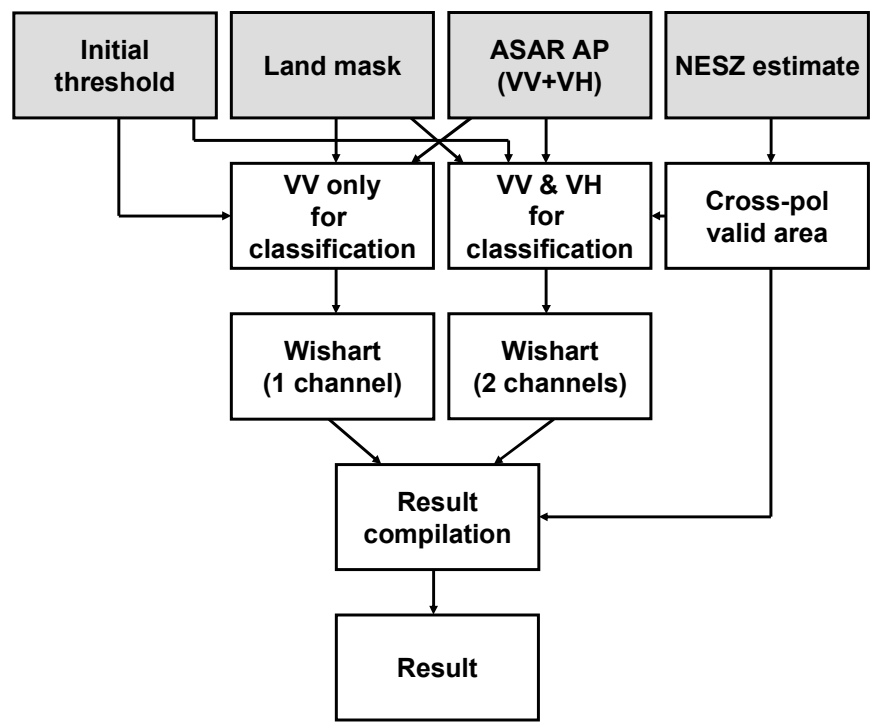

Figure 5. Classification concept (step 1) with limited use of crosspolarization information. Step 2 applies the same concept to split one class.
Ice charts suggest only two old ice types with some grey ice present in the scene. A four class approach does not work appropriately; the initialization of the classifier with three classes is therefore suggested in this case. The initial classes are set up by first using the median value of the marine area for the crosspol channel to separate low and high backscatter pixels. The pixels with high cross-pol level are further separated using the median co-pol value.

The Bayesian classifier is run with three iterations, where the class means are updated after each iteration. Both classifiers (single-pol and dual-pol) use the same class information for the update. Only the marine areas (open water and sea ice) are included in the process.

The fourth class is determined in a second step which is described in more detail in the next section. The interpretation of the final classes is a manual task; however, some assumptions can be made based on backscatter levels.

\section{Discussion OF RESUlts}

Fig. 6 shows four step 1 classification results, all of which were derived using different methods. The cross-pol information only was used to generate Fig. 6a. The NESZ variation clearly affects the result in far range, particularly in the lower half of the image. Co-pol information only was used in the case of Fig. 6b. Wind roughening of the water surface is the main cause for confusion of sea ice and open water.

Fig. 6c uses the dual polarization information for classification. It is apparent that the extra noise in far range ( $\mathrm{VH}$ level increase) has a negative effect on the result.

The result of the new approach is shown in Fig. 6d. In far range the result is visually improved, although some class confusion remains. The reason for the latter is that both $\mathrm{VH}$ and $\mathrm{VV}$ show potential for confusion in far range. In the case of $\mathrm{VH}$, the effect is system related, whereas VV is sensitive to a wind roughened water surface. Grey ice is not identified.

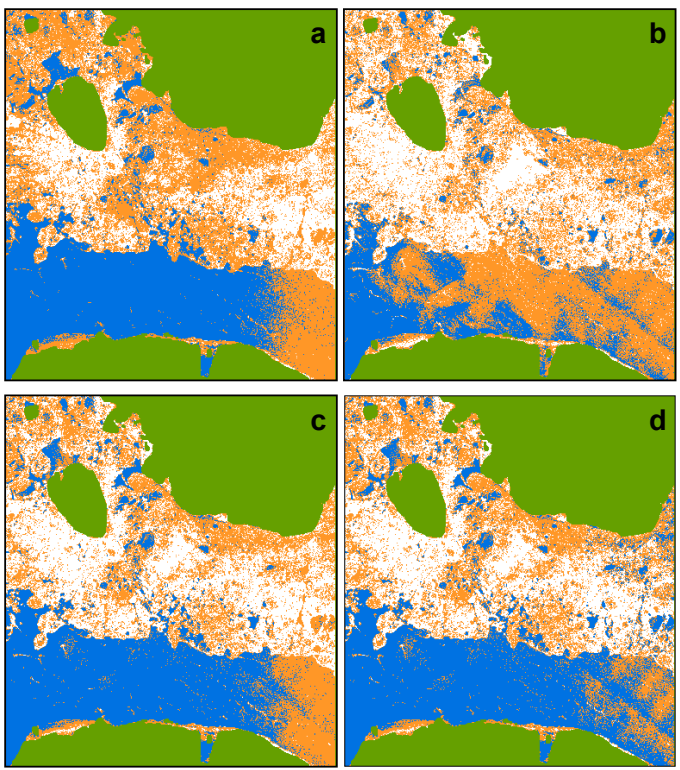

Figure 6. Classification results (step 1) based on different inputs: a) VH only; b) VV only; c) $\mathrm{VV}+\mathrm{VH}$, d) $\mathrm{VV}+$ spatially restricted $\mathrm{VH}$. 


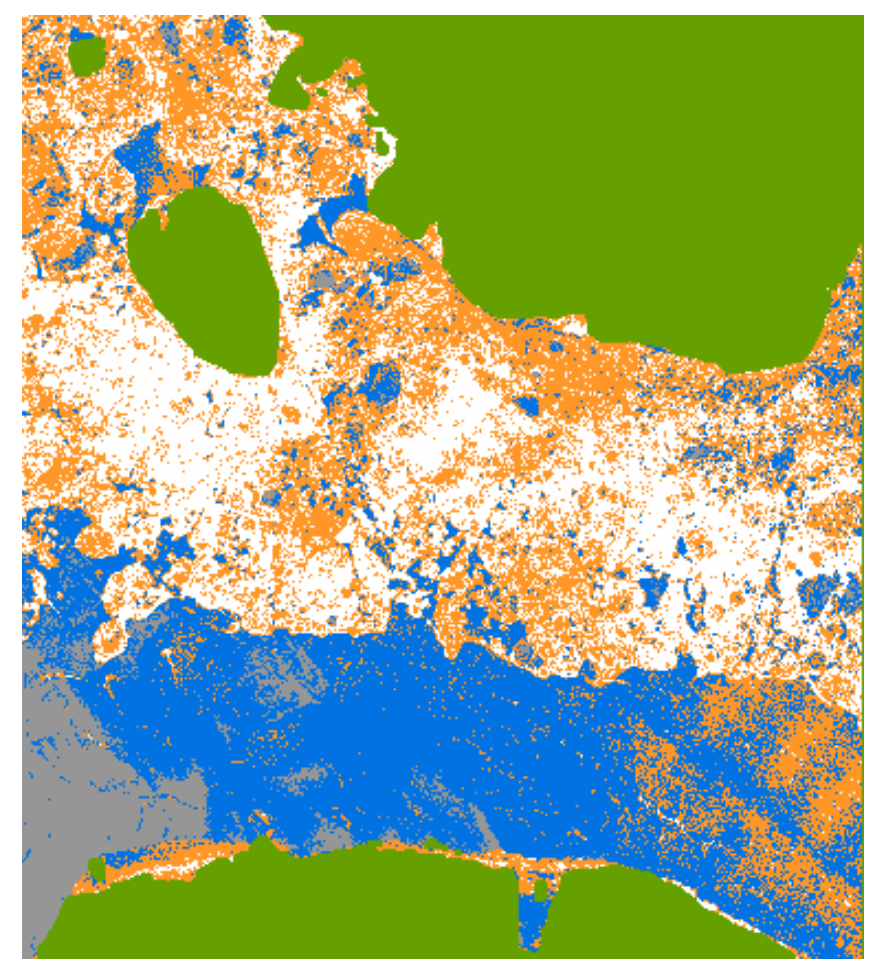

Figure 7. Final classification result based on a two step approach.

None of the results identifies the grey ice in the scene. A second step in the classification is therefore applied. In this step only the blue class from Fig. $6 \mathrm{~d}$ (lowest backscatter) is considered and divided into two classes thus adding an additional class to the final result.

Table II summarizes the backscatter statistics for the classes of the final result shown in Fig. 7. The mean cross-pol level for all four classes is close to or below the worst case NESZ of $-19 \mathrm{~dB}$ posted for this swath. Compared to the NESZ estimate shown in Fig. 2, all classes are above this level for an incidence angle range between $31^{\circ}$ and $34.5^{\circ}$.

Grey ice (class 4) shows little difference to open water (class 3) for VH but is markedly different in VV. VH provides more contrast than VV between classes 2 and 3 .

\section{CONCLUSIONS}

ENVISAT ASAR is the first SAR satellite equipped with dual polarization modes. Higher information content is expected from two channels; however, the system noise level and the variation of the NESZ are of concern for low backscatter situations. Cross-polarization sea ice signatures are usually low, which may result in an SNR that is close to or even below zero.
TABLE II. Class AveragES

\begin{tabular}{|l|r|r|r|r|c|}
\hline \multirow{2}{*}{ Class } & \multicolumn{5}{|c|}{ Class average information } \\
\cline { 2 - 6 } & $\begin{array}{c}\text { VV } \\
\text { mean }\end{array}$ & VV std & $\begin{array}{c}\text { VH } \\
\text { mean }\end{array}$ & VH std & $\begin{array}{c}\text { color } \\
\text { assignment }\end{array}$ \\
\hline Class 1 & -9.28 & 0.90 & -18.48 & 1.15 & white \\
\hline Class 2 & -10.99 & 0.77 & -20.40 & 1.00 & orange \\
\hline Class 3 & -12.62 & 0.89 & -22.95 & 1.26 & blue \\
\hline Class 4 & -18.47 & 2.87 & -23.89 & 0.80 & gray \\
\hline
\end{tabular}

A novel classification scheme was introduced to deal with cases where the SNR is low in the cross-pol channel. The scheme allows the use of cross-polarization in combination with co-polarization information in areas where a positive SNR is expected and utilizes co-pol information only otherwise. Comparisons to single polarization classifications and the standard dual polarization classification indicate an improvement for the new method.

An additional second step is applied to separate open water and grey ice. This approach is successful for this scene due to environmental conditions. Open water is wind roughened thus causing increased backscatter compared to grey ice. The result of the second step should therefore not be generalized.

Average cross-polarization backscatter levels for all classes are reported at or below the posted worst case NESZ for the swath. The proposed method allows the utilization of the cross-polarization channel in a large portion of the image. Detailed NESZ information, in particular its variation over range, is required for successful classification.

\section{ACKNOWLEDGMENTS}

ENVISAT ASAR data were provided by the European Space Agency to CIS under AO Project ID100. Meteorological data was provided by Environment Canada. The authors would like to thank Dean Flett and Roger De Abreu from CIS for valuable discussions.

\section{REFERENCES}

[1] B. Scheuchl, R. Caves, D. Flett, R. De Abreu, M.Arkett, and I. G. Cumming, "Potential of Cross-Polarization Information for Operational Sea Ice Monitoring". In Proc. ENVISAT/ERS Symposium, Salzburg, Austria, September 6-10, 2004.

[2] S.V. Nghiem, and C. Bertoia, "Study of multi-polarization C-band backscatter signatures for Arctic sea ice mapping with future satellite SAR," Canadian Journal of Remote Sensing, vol. 27, no. 5, October, 2001.

[3] J.S. Lee, M.R. Grunes, E. Pottier, "Quantitative comparison of classification capability: fully polarimetric versus dual and single-polarization SAR," IEEE Transactions on Geoscience and Remote Sensing, Volume: 39, Issue: 11, Nov. 2001, Pages: $2343-2351$. 